\title{
Neutrino research program with Nuclear Emulsion at J-PARC
}

\section{Tsutomu Fukuda*t}

Nagoya University

E-mail: tfukuda@flab.phys.nagoya-u.ac.jp

\begin{abstract}
We proposed a new experimental project which equips Emulsion Cloud Chamber (ECC) as a main detector in order to study low energy neutrino-nucleus interactions in detail and explore a possible existence of sterile neutrinos. First of all, a test experiment (J-PARC T60) is implemented to check the performance of newly developed emulsion gel, optimize the detector structure, and demonstrate the neutrino analysis with ultimate position resolution. Anti neutrino beam was exposed to a few $\mathrm{kg}$ iron and water target ECC and a $60 \mathrm{~kg}$ iron target ECC at J-PARC. From last summer, the analysis based on several thousands of anti-neutrino interactions accumulated in ECC was started. In this paper, the status of the measurements and the future prospects will be given.
\end{abstract}

The 3rd International Symposium on "Quest for the Origin of Particles and the Universe" 5-7 January 2017

Nagoya University, Japan

\footnotetext{
* Speaker.

${ }^{\dagger}$ on behalf of the NINJA Collaboration
} 


\section{Introduction}

The cross section measurements of neutrino-nucleus interactions in sub-multi $\mathrm{GeV}$ region is important to reduce the systematic uncertainty of the current and future neutrino oscillation experiments for finding the $\mathrm{CP}$ violating phase in lepton sector and decision the mass ordering of neutrinos [1, 2, 3, 4, 5, 6, 7, 8, 9]. While, one possible explanation of LSND [10] and MiniBooNE [11] anomaly is a result of the existence of sterile neutrino. The MiniBooNE anomaly was detected as an excess of the electron neutrino events in sub $\mathrm{GeV}$ region. So the precise measurement of electron neutrino interaction is important. The nuclear emulsion detector is suitable to investigate neutrino interactions in detail with its ultimate position resolution. We are implementing a test experiment for this purpose in J-PARC, so-called J-PARC T60 experiment. In this paper, the experimental concept, the analysis status and future prospects of this experimental program is described.

\section{Conceptual design for the detectors}

Nuclear emulsion is a three-demensional solid tracking detector with sub-micron resolution. Thanks to its high spatial resolution, we can investigate the event feature in detail, for example slow proton (less than several tens of $\mathrm{MeV}$ ) are detected at high efficiency [12], and also separate an electron-neutrino charged current interaction from main background source of a muon-neutrino neutral current interaction with $\pi^{0}$ production very well [13]. The detector using nuclear emulsion has a lot of flexibility for target material selection because the detector, so-called Emulsion Cloud Chamber (ECC), is constructed as a sandwich structure of thin nuclear emulsion films and the target material, even if water is available for the target in principle.

The ECC technique has a proton identification capability from other high energy charged particles using $\mathrm{dE} / \mathrm{dx}$, momentum measurements [14]. However, $\pi / \mu$ separation at more than sub$\mathrm{GeV}$ in ECC is difficult, except in the very huge emulsion detector as the OPERA detector [15]. So in this time, we use one of near detectors in the T2K experiment, INGRID [16] to identify the muon tracks. The tracking device of INGRID is a 1-cm-thickness plastic scintillator $(5 \mathrm{~cm}$ width) read out with wave length-shifting fibers. The constitution of the detector is a sandwich structure of the tracking planes and 6.5-cm-thickness iron blocks. The detector was assembled from a total of nine iron layers $(58.5 \mathrm{~cm})$ and 11 vertical and horizontal tracking planes. Furthermore, we set the emulsion shifter [17] between the ECC and the INGRID in order to connect tracks using timing matching method in this time. The emulsion shifter has the mechanically controlled moving stages with different frequency and give a timing information by track coincidence between the films on the moving stages. Fig. 1 1 shows the schematic view of the detector setup.

\section{The quality of newly developed emulsion gel}

Nuclear emulsion gel was produced at the facility of Nagoya University. In this time, highsensitive emulsion gels, containing $55 \%$ or $45 \% \mathrm{AgBr}$ crystal by volume ratio was produced. Both of newly developed emulsion gels were investigated the sensitivity and the noise accumulating for half of year and were confirmed to be kept at safety level for the physics analysis. Two emulsion layers, each 50-60 $\mu \mathrm{m}$ thick, were formed on both faces of a 180- $\mu \mathrm{m}$ thick polystyrene plate. 


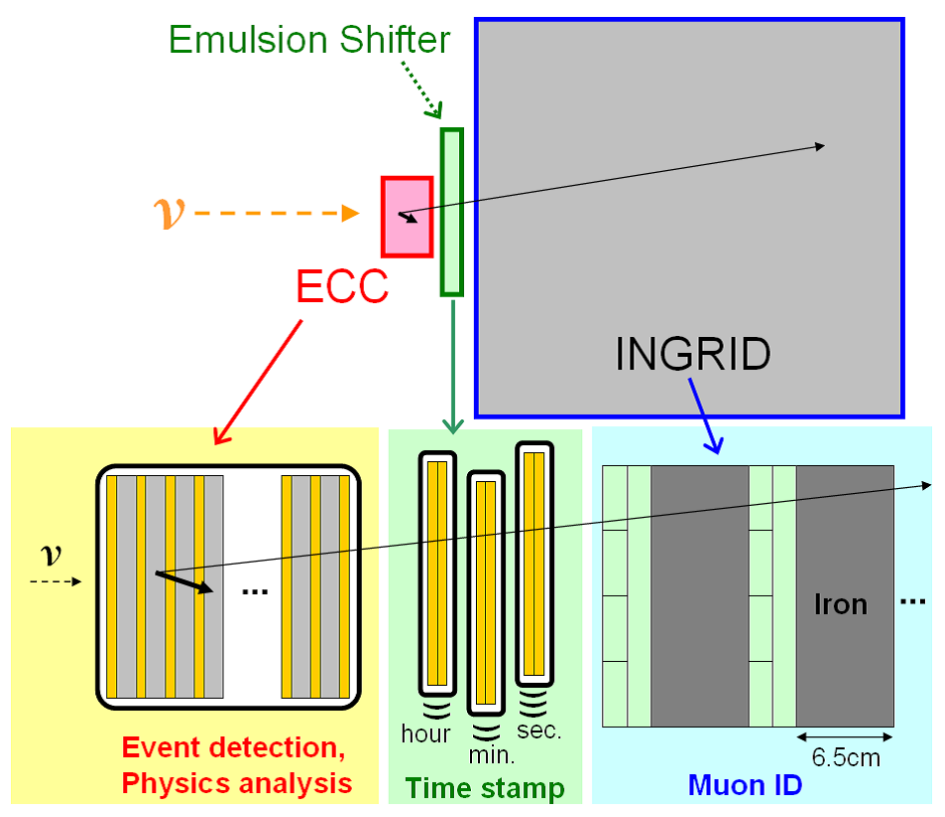

Figure 1: Detector concept

\section{Neutrino beam exposure}

So far, three times anti-neutrino beam exposure were finished in the J-PARC T60 experiment. The exposure period, beam mode, accumulated proton on target (POT), target material and mass are shown in Table. 1 .

Table 1: Status of $v$ beam exposure

\begin{tabular}{|c||c||c||c||c||c|}
\hline Exposure & Period & Beam mode & POT & Target & Mass $(\mathrm{kg})$ \\
\hline 1 & Jan. - Apr. 2015 & anti-neutrino & $1.38 \times 10^{20}$ & iron & 2 \\
2 & May - Jun. 2015 & anti-neutrino & $0.8 \times 10^{20}$ & water & 1 \\
3 & Jan. - May 2016 & anti-neutrino & $3.95 \times 10^{20}$ & iron & 60 \\
\hline
\end{tabular}

The aim of the exposure.1 is the feasibility study. We checked the quality of newly developed emulsion gel, the handling of emulsion detector at J-PARC site and the demonstration of neutrino event analysis. In the exposure.2, we tried to product a water target emulsion detector and checked the detector performance for the first time. Then the exposure. 3 was carried out in order to confirm the detector performance with high statistics of neutrino interactions. These test experiments ran parasitically with the $\mathrm{T} 2 \mathrm{~K}$ experiment. So the beam mode was fixed to the anti-neutrino beam mode. The $30 \%$ of all interaction is neutrino interaction, not anti-neutrino interaction, because there are contamination of neutrinos in the anti-neutrino beam. The detectors were placed about $1 \mathrm{~m}$ from the neutrino beam center. The neutrino energy flux is widely distributed from sub-GeV to a few $\mathrm{GeV}$ in this location. The total expected number of neutrino and anti-neutrino events are $\sim 20, \sim 5$ and $\sim 3000$ fiducial volume events in exposure.1, 2 and 3 respectively. The picture of the detectors in exposure.1, 2 and 3 is shown in Fig, 2 , and 4 

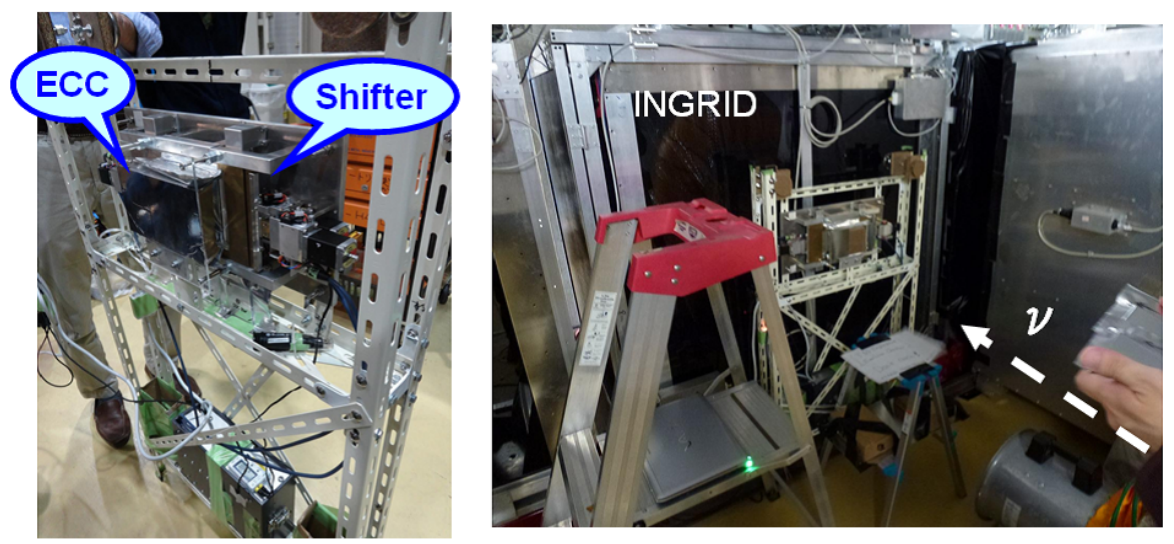

Figure 2: Detector in exposure.1

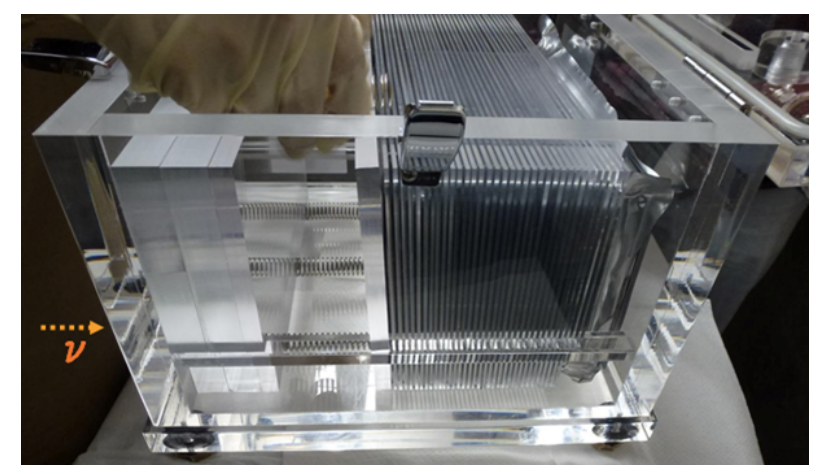

Figure 3: Detector in exposure.2

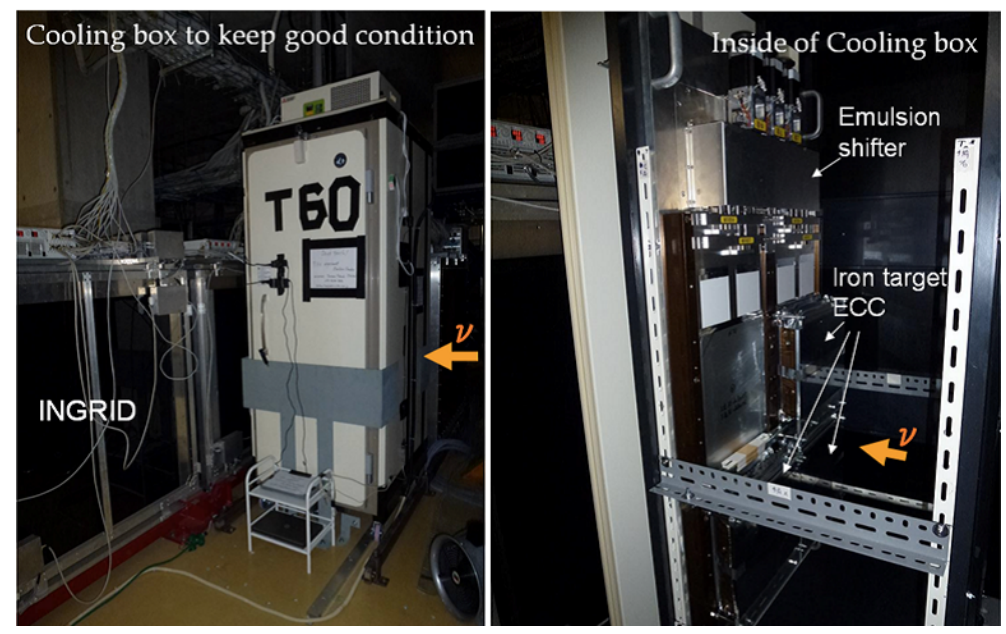

Figure 4: Detector in exposure.3 


\section{Analysis status}

\subsection{Exposure.1}

The ECC consisted of the emulsion films and $500 \mu \mathrm{m}$ thickness iron plates. After development, the emulsion films were scanned by the high-speed automatic emulsion scanning system, HTS [18] at Nagoya Univ. Then the tracks were reconstructed and neutrino event vertex was searched. Consequently, more than 10 neutrino events were detected as shown in Fig. 5] and more than 100 electron/positron pair vertices from gamma-ray were also detected. The proton-like tracks were found with $\mathrm{dE} / \mathrm{dx}$ and momentum measurement as shown in Fig. 6 The long-term operation of the emulsion shifter was also confirmed on the site by checking the position repeatability of each stages. It was confirmed less than $1 \mu \mathrm{m}$ and enough to use. Then the timing resolution is approximately 7.9 seconds, which was investigated using tracks passing through the emulsion shifter and INGRID. Finally, the hybrid analysis with INGRID was successfully demonstrated as shown in Fig. 7 The details of analysis is described at [19, 20]

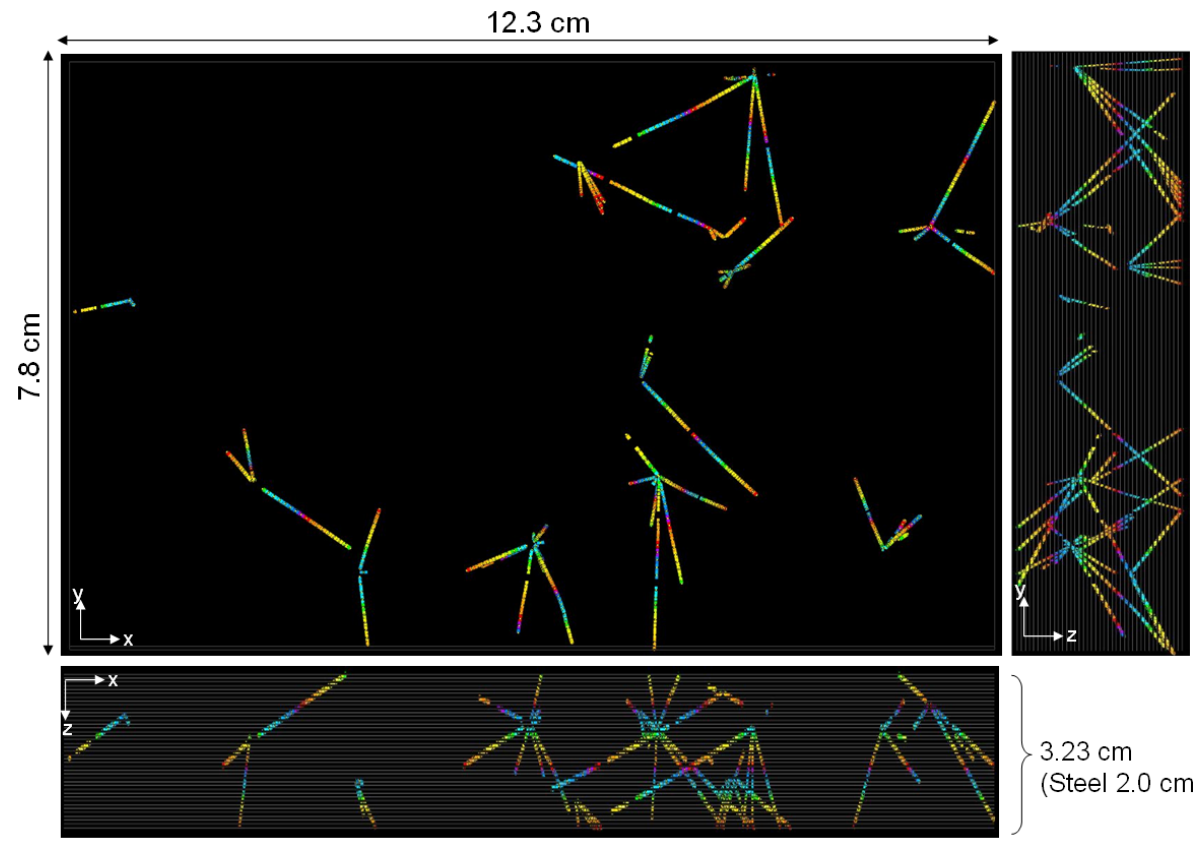

Figure 5: The detected neutrino events

\subsection{Exposure.2}

The detector is the sandwich structure of the vacuum packed emulsion films and the 2.0-mmthickness frame type acrylic spacers to fill the water in the space. In this time, $250 \mu \mathrm{m}$ thickness iron plates or $300 \mu \mathrm{m}$ or $400 \mu \mathrm{m}$ thickness acrylic plates are packed with emulsion films as a support. As a result, one neutrino-water interaction was detected as shown in Fig. 8, However, we also found large distortion of films in each structure. The standard deviation of the distance between the films are measured at a few hundred $\mu \mathrm{m}$ level. That makes difficult to reconstruct tracks in the emulsion analysis. So we need to select much stronger support from the next time. 


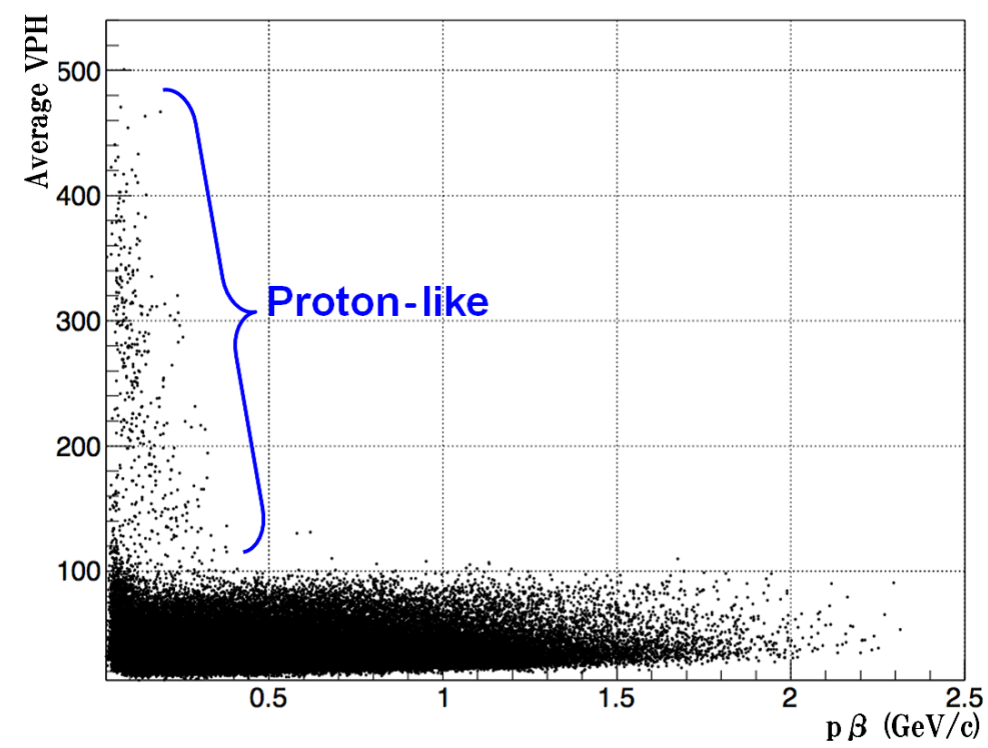

Figure 6: Proton identification. The horizontal and vertical axis shows the momentum and $\mathrm{dE} / \mathrm{dx}$ for each tracks. In ECC, dE/dx is measured as a track blackness, so-called Average VPH and the momentum is measured by Multiple Coulomb Scattering method.
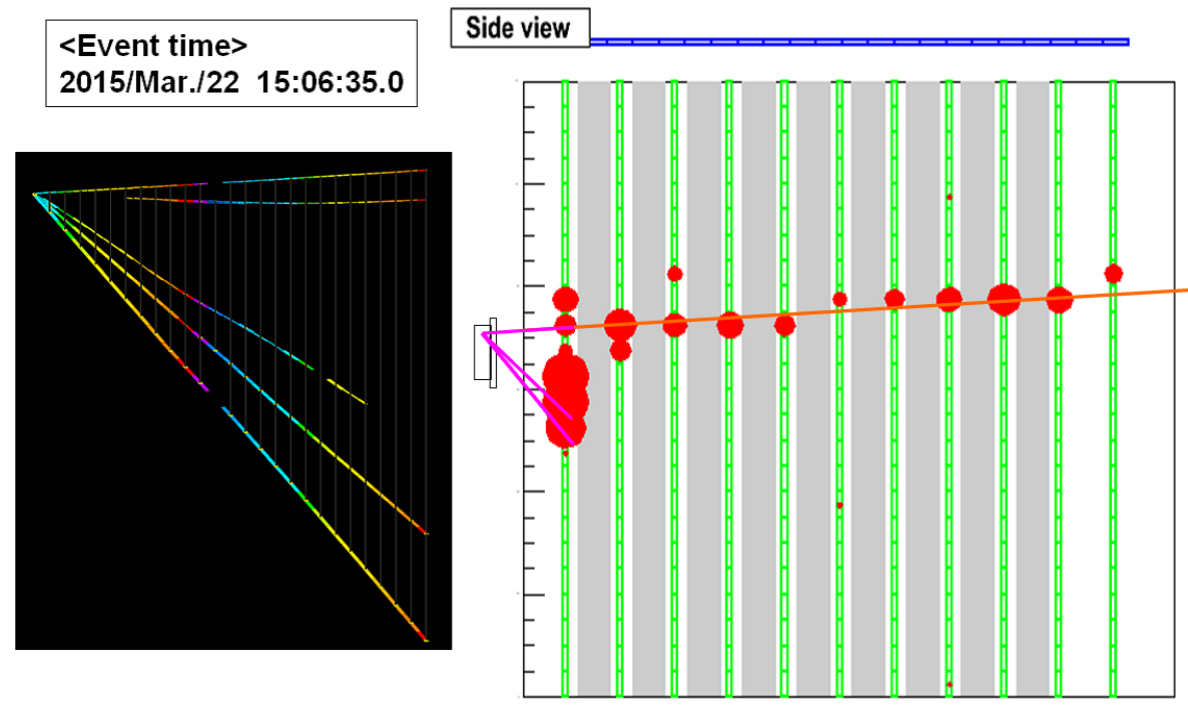

Figure 7: Hybrid analysis. The event topology is well matched between ECC and INGRID. 


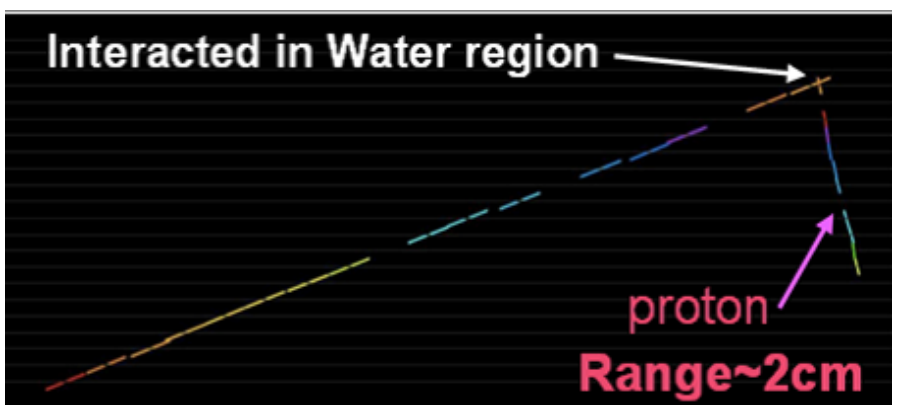

Figure 8: First detection of $v$-water interaction in the emulsion detector.

\subsection{Exposure.3}

In the exposure.3, the $500 \mu \mathrm{m}$ thickness iron plates and emulsion films were used for the ECC and a large size emulsion shifter was used and operated well for the beam exposure. Fig. 9 shows the history of the detector preparation, the beam exposure, the hardware treatments before scanning and the film scanning. The film scanning was done with high tracking efficiency (95-98\%) and the analysis is now going on. Currently, more than 100 neutrino candidate events were already detected in a small part of the ECC analysis.

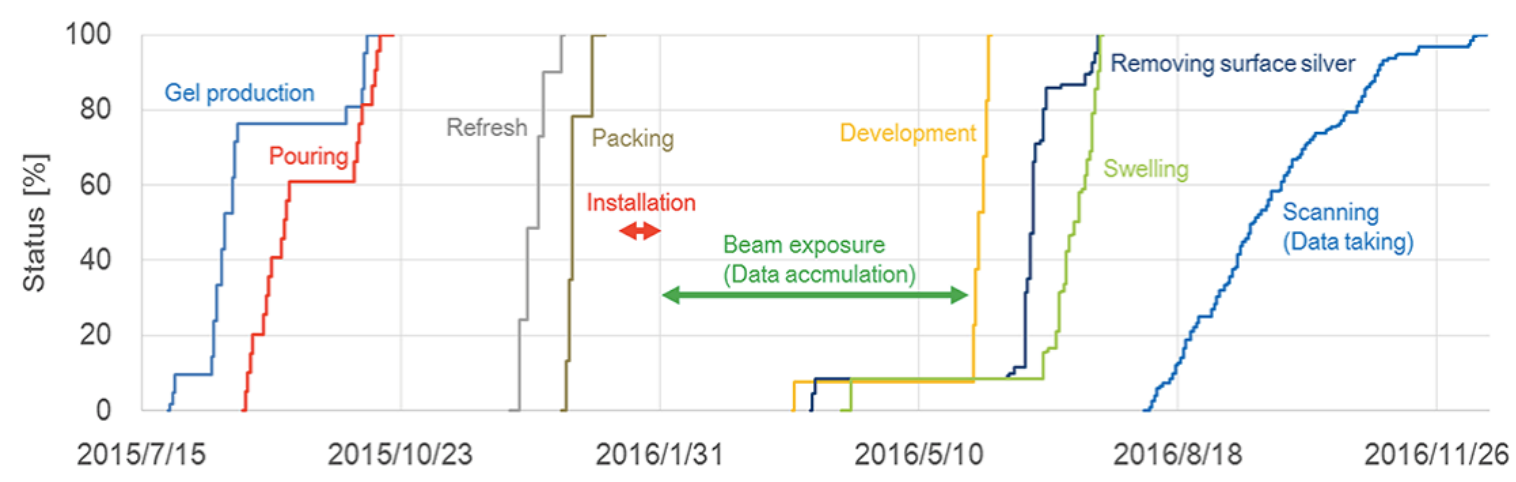

Figure 9: History of the exposure.3

\section{Future prospects}

We plan to expose neutrino beam to a $100 \mathrm{~kg}$ scale water target ECC from the latter half of 2018 in order to measure the neutrino-water cross-section with high statistics. Toward this purpose, we will confirm the detector performance and establish the analysis method by analyzing $60 \mathrm{~kg}$ iron target ECC of the exposure.3 in detail. Also, R\&D for the water target ECC has been carried out from last December to confirm the support plate and finally optimize the detector setup of the large size water target ECC.

After the detailed study for neutrino-nucleus interactions including the measurement of the electron neutrino cross-section, we will propose an experiment to explore sterile neutrinos with 
ton-scale emulsion detector around 2020. In this experiment, a near detector is set at first and then a far detector will be installed to reduce the systematic uncertainty at $1 \mathrm{~km}$ from the proton target.

Furthermore, we may have a chance to search for a charmed-penta quark (uudd $\bar{c}$ or $u u d s \bar{c}$ ) [21] or a charmed nuclei [22] at near the production threshold energy of charmed particles with low energy neutrino/anti-neutrino beam. The discussion for such possibility is now in progress.

\section{Conclusions}

We are implementing an experimental project for the precise measurement of neutrino-nucleus interactions for the neutrino oscillation physics with nuclear emulsion detector at J-PARC. The J-PARC T60 experiment is a first step of this project in order to make the coming large scale experimental plan. We actually demonstrated the neutrino analysis in the iron and water target ECC and a hybrid analysis with the T2K near detector at J-PARC. We will expand the detector scale gradually and there is promise to obtain the important/unique results within several years.

\section{References}

[1] K. Abe et al. (T2K Collaboration), Phys. Rev. D 88, (2013) 032002.

[2] K. Abe et al. (Hyper-Kamiokande Proto-Collaboration), PTEP 2015 (2015) $053 \mathrm{C} 02$.

[3] P. Adamson et al. (NovA Collaboration), Phys. Rev. Lett. 116 (2016) 151806.

[4] R. Acciarri at al. (DUNE Collaboration), arXiv:1512.06148 [physics.ins-det].

[5] F. An et al. (JUNO Collaboration), arXiv:1507.05613 [physics.ins-det].

[6] S. B. Kim, arXiv:1504.08268 [physics.ins-det].

[7] M. Athar et al. (INO Collaboration), http://www.imsc.res.in/ ino/OpenReports/INOReport.pdf.

[8] M. G. Aartsen et al. (IceCube PINGU Collaboration), arXiv:1401.2046 [physics.ins-det].

[9] S. Adrian-Martinez et al. (KM3NeT Collaboration), arXiv:1601.07459 [astro-ph.IM].

[10] A. Aguilar et al. (LSND Collaboration), Phys. Rev. D 64 (2001) 112007.

[11] A. A. Aguilar-Arevalo et al. (MiniBooNE Collaboration), Phys. Rev. Lett. 110 (2013) 161801.

[12] T. Fukuda et al., JINST 8 (2013) P01023.

[13] N. Agafonova et al. (OPERA Collaboration), JHEP 07 (2013) 004.

[14] T. Toshito et al., Nucl. Instrum. Meth. A 516 (2004) 436.

[15] T. Fukuda for the OPERA Collaboration, Nuovo Cim. C 39 (2017) 315.

[16] K. Abe et al. (T2K Collaboration), Nucl. Instrum. Meth. A 694 (2012) 211.

[17] H. Rokujo et al., Nucl. Instrum. Meth. A 701 (2013) 127.

[18] M. Yoshimoto et al., arXiv:1704.06814 [physics.ins-det].

[19] T. Fukuda et al., arXiv:1703.03659 [physics.ins-det].

[20] K. Yamada et al., arXiv:1703.03737 [physics.ins-det].

[21] G. De Lellis et al., Nucl. Phys. B 763 (2007) 268.

[22] G. Onengut et al. (CHORUS Collaboration), Nucl. Phys. B 718 (2005) 35. 